\title{
Relationship between vascular endothelial growth factor and macular edema in retinal vein branch obstruction
}

This article was published in the following Dove Press journal:

Clinical Ophthalmology

\section{Hideaki Okawada \\ Yuta Saito \\ Shigehiro Iwabuchi \\ Shotaro Kosuge \\ Haruo Takahashi \\ Hidetoshi Onda \\ Masahiro Kito}

Department of Ophthalmology, Showa University, Tokyo, Japan
Correspondence: Hideaki Okawada Department of Ophthalmology, Showa University, 2-I-16 Nishi-nakanobu, Shinagawa-ku, Tokyo 142-0054, Japan Email okawada I 107@yahoo.co.jp
Purpose: Vascular endothelial growth factor (VEGF) plays an important role in branch retinal vein occlusion (BRVO) with cystoid macular edema (CME). Monitoring changes in VEGF is crucial for evaluating treatment but requires vitreous or aqueous humor sampling, which hampers its clinical application. We investigated the correlation between VEGF and protein concentration in the aqueous humor (flare) and whether this could be used to monitor treatmentrelated VEGF changes.

Design: This retrospective observational study involved 19 previously untreated patients with BRVO. Aqueous humor was obtained, and intravitreal ranibizumab (IVR) injection was administered to these patients. The correlation between VEGF and flare, central retinal thickness (CRT), and best-corrected visual acuity (BCVA) was investigated. Differences in these values were considered between pre-IVR and 1 week and 1-3 months post-IVR. Moreover, in patients with recurrence who received additional IVR, further changes in VEGF were examined.

Main outcome measures: The end point of this study was BCVA, flare, and CRT at the fovea.

Results: Significant improvement was seen in BCVA and CRT at all time points and in Flare at 1 vs 3 months post-IVR; nevertheless, additional IVR was necessary in $94 \%$ of cases. In a patient with recurrence, CRT did not improve, even though VEGF decreased.

Conclusion: Flare may be effective for estimating VEGF levels in aqueous humor pre-IVR. Inflammation-related molecules other than VEGF may be related to recurrence.

Keywords: aqueous humor, branch retinal vein occlusion, flare, protein density, ranibizumab, vascular endothelial growth factor

\section{Introduction}

Vascular endothelial growth factor (VEGF) is an important factor in branch retinal vein occlusion (BRVO) with cystoid macular edema (CME). Anti-VEGF therapy is widely used as the first-line treatment for inhibiting its progression. Monitoring changes in VEGF is important as a means of evaluating the effect of treatment, but this requires sampling of vitreous or aqueous humor, and this approach is difficult to use in the clinic.

Miyake et $\mathrm{al}^{1}$ reported that an increase in protein concentrations (flare) in the aqueous humor of the anterior chamber is an index of inflammation in BRVO. We therefore investigated whether flare and VEGF in the aqueous humor of patients with BRVO are correlated and whether this can be used to estimate VEGF levels. Additionally, we used this approach to assess changes in VEGF after anti-VEGF therapy and with CME recurrence. 


\section{Methods}

This was a retrospective observational study. Nineteen patients with BRVO who attended our hospital from March 2014 to December 2014 were enrolled. Intravitreal ranibizumab (IVR) is administered to all such patients in this hospital. Patients who had previously received steroid injection into the vitreous humor, or Tenon capsule, anti-VEGF therapy (eg, ranibizumab, bevacizumab, and aflibercept), retinal photocoagulation, or vitrectomy, were excluded. This study was approved by the Medicine Bio-Ethical Committee of Showa University School. Although this study is a retrospective study, it is done with patient consent before the study based on the guidelines of our ethics committee. We got written permission to collect specimens from the eye and do conference presentations and paper preparation based on that data.

The end point of this study was best-corrected visual acuity (BCVA), flare, and central retinal thickness (CRT) at the fovea. BCVA was converted into LogMAR units after measurement with a Landolt ring. Flare was measured using an FC-2000 flare cell meter (Kowa, Nagoya, Japan), and CRT was measured using a CIRRUS HD-OCT (Carl Zeiss Meditec AG, Jena, Germany).

Paracentesis of the aqueous humor was performed to adjust intraocular pressure immediately prior to IVR, and aqueous humor was collected on this occasion. The collected aqueous humor was stored with freezing at $-80^{\circ} \mathrm{C}$. VEGF levels in the aqueous humor samples were subsequently measured using enzyme-linked immunosorbent assays (R\&D Systems, Inc., Minneapolis, MN, USA). BCVA, flare, and CRT were measured preoperatively and 1 week and 1, 2, and 3 months postoperatively.

If CRT was $>300 \mu \mathrm{m}$ at 3 months after injection, additional IVR or additional IVR + laser were performed. Laser treatment was adapted as per the Branch Vein Occlusion Study. ${ }^{2}$ The correlation of VEGF with BCVA, flare, and CRT was determined.

Data were expressed as mean \pm standard deviation, and differences were considered statistically significant if $P$-values were $<0.05$. To compare BCVA, flare, and CRT data preoperatively and at 1 week and 1, 2, and 3 months postoperatively, the Pearson's correlation $t$-test was used. Pearson's correlation coefficient and the recurrence function were used to assess the correlation of VEGF with BCVA, flare, and CRT.

\section{Results}

The data of the BRVO group are listed in Table 1. Preoperatively, VEGF correlated significantly with flare (Figure 1
Table I Characteristics of the BRVO before intravitreal ranibizumab administration

\begin{tabular}{ll}
\hline Comparison item & BRVO $(\mathbf{n}=1 \mathbf{9})$ \\
\hline Age (years) & $71.05 \pm 9.05$ \\
Sex distribution & $M 13, \mathrm{~F} 6$ \\
BCVA (LogMAR) & $0.52 \pm 0.48$ \\
Flare $(\mathrm{pc} / \mathrm{ms})$ & $8.19 \pm 3.61$ \\
VEGF $(\mathrm{pg} / \mathrm{mL})$ & $188.78 \pm 219.91$ \\
CRT $(\mu \mathrm{m})$ & $509.26 \pm 130.93$ \\
\hline
\end{tabular}

Abbreviations: BCVA, best-corrected visual acuity; BRVO, branch retinal vein occlusion; CRT, central retinal thickness; $F$, female; $M$, male.

and Table 2). However, there was no significant correlation between VEGF and BCVA or CRT.

The changes in BCVA, flare, and CRT after IVR are shown in Figures 2-4. When each of these items was compared before and after IVR, the improvement was significant for BCVA and CRT at all time points and in flare for 1 vs 3 months after IVR.

Fifteen of the 16 patients with BRVO suffered recurrence, as determined at the 3 months' follow-up after IVR. Additional IVR + Laser was used to treat 11 patients, and additional IVR only was used to treat four patients with recurrent disease. Among the patients with recurrence, aqueous humor was again obtained from seven patients prior to additional IVR administration. The changes in VEGF, CRT, and flare values are shown in Table 3. There was no significant correlation between VEGF and CRT or flare at the time of additional IVR treatment (Table 4).

\section{Discussion}

There was a correlation between VEGF and flare before IVR. In the report of Miyake et al, ${ }^{1}$ there was an increased

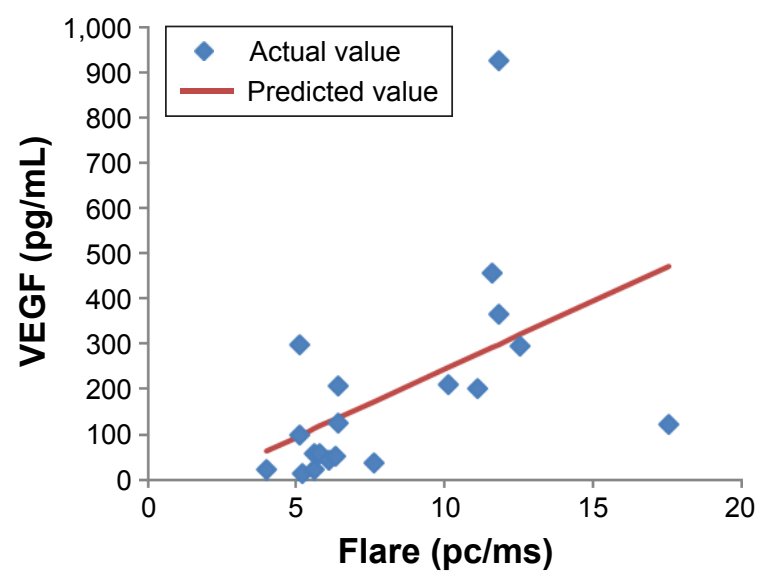

Figure I A correlation between VEGF concentration and flare preoperatively, as shown by the single regression line $(y=30.3 x-59.1)$.

Abbreviation: VEGF, vascular endothelial growth factor 
Table 2 Correlation between VEGF and flare, CRT, and BCVA prior to intravitreal ranibizumab administration

\begin{tabular}{lll}
\hline $\begin{array}{l}\text { Item of } \\
\text { correlation }\end{array}$ & $\begin{array}{l}\text { Coefficient of } \\
\text { correlation with VEGF }\end{array}$ & P-value \\
\hline Flare & 0.5 & 0.03 \\
CRT & 0.11 & 0.65 \\
BCVA & 0.4 & 0.09 \\
\hline
\end{tabular}

Abbreviations: $\mathrm{BCVA}$, best-corrected visual acuity; CRT, central retinal thickness; VEGF, vascular endothelial growth factor.

inflammatory response in BRVO. In addition, in the report of Noma et al, ${ }^{3,4}$ VEGF was shown to correlate with IL-6. Although flare may reflect inflammation rather than increased VEGF, flare may also be useful for predicting VEGF prior to IVR.

When each item was compared before and after IVR, there was a significant improvement in BCVA and CRT from 1 week post-IVR. In addition, there was a significant difference in flare values from 1 month post-IVR. However, the time course of CRT changes indicated a tendency for deterioration over a period of 2-3 months and the relapse rate at 3 months was $94 \%$. In the BRAVO study, ${ }^{5}$ it was necessary to use 2.4-fold higher dosage by 6 months after the shift to PRN. Ito et $\mathrm{al}^{6}$ used 1.1 times additional dosage by 1 year after shifting to PRN. It therefore seems that it is difficult to prevent the recurrence of CME by a single IVR administration.

Among the seven patients in whom VEGF levels were examined at the time of additional IVR treatment, one patient (case 7) showed markedly improved VEGF, CRT, and flare at the time of additional IVR administration, as compared with initial values, but in other patients (cases 1, 3, and 5), CRT had returned to pre-IVR levels and VEGF values were worse than before IVR, even though flare had improved. Thus, multiple IVR treatments should be considered if the initial VEGF level is high. As there was no significant correlation between VEGF and CRT after recurrence in this

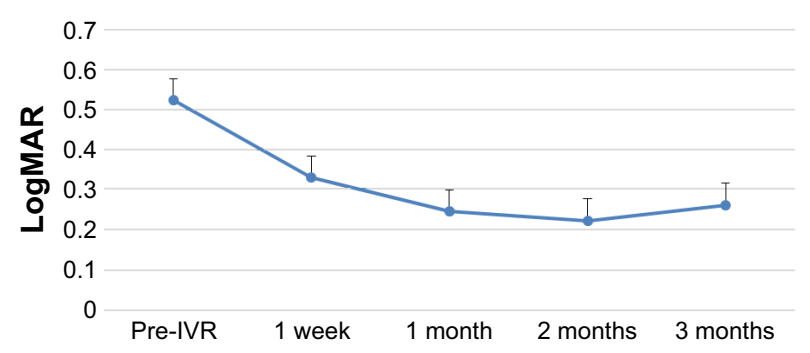

Figure 2 Time course of BCVA changes after IVR.

Note: The improvement was significant for all time points after IVR, as compared with before IVR.

Abbreviations: BCVA, best-corrected visual acuity; IVR, intravitreal ranibizumab.

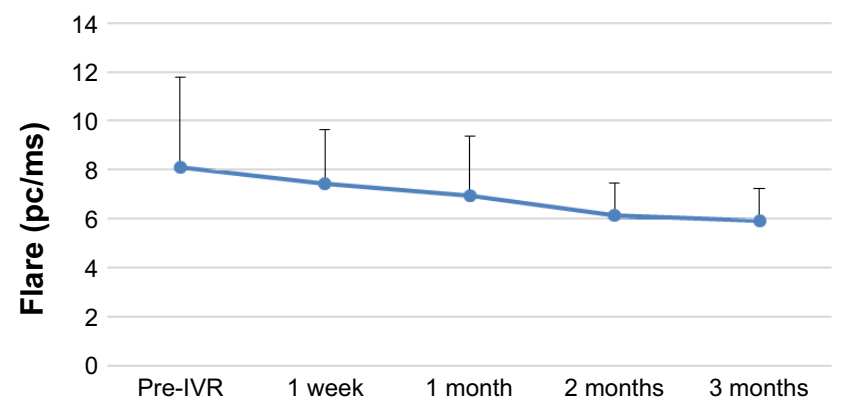

Figure 3 Time course of flare changes after IVR treatment.

Note: The improvement was significant for all time points after IVR, as compared with before IVR.

Abbreviation: IVR, intravitreal ranibizumab

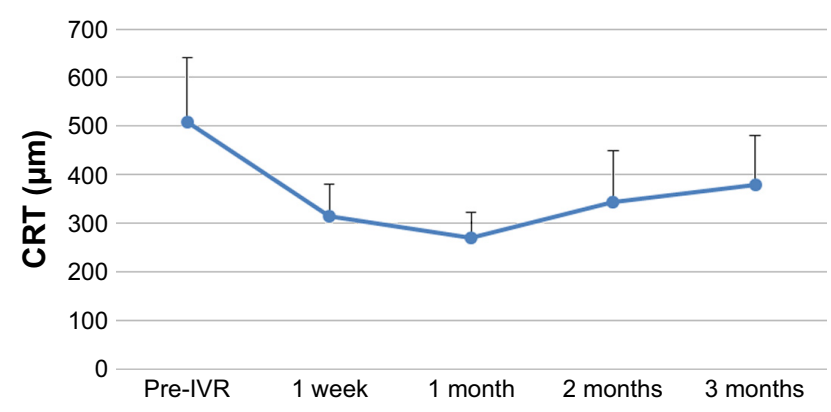

Figure 4 Time course of changes in CRT at the fovea after IVR treatment.

Note: The improvement was significant for all time points after IVR, as compared with before IVR.

Abbreviations: CRT, central retinal thickness; IVR, intravitreal ranibizumab.

Table 3 The changes in VEGF, CRT, and flare values at the additional IVR

\begin{tabular}{|c|c|c|c|c|c|c|}
\hline & \multicolumn{2}{|c|}{ VEGF $(\mathrm{pg} / \mathrm{mL})$} & \multicolumn{2}{|c|}{ CRT $(\mu \mathrm{m})$} & \multicolumn{2}{|c|}{ Flare (pc/ms) } \\
\hline & Pre & Post & Pre & Post & Pre & Post \\
\hline Case I & 22.18 & 26.85 & 574 & 438 & 5.6 & 5.4 \\
\hline Case 2 & 456.87 & 391.43 & 390 & 535 & 11.6 & 8.7 \\
\hline Case 3 & 96.96 & 148.38 & 460 & 452 & 5.1 & 5.4 \\
\hline Case 4 & 36.2 & 54.89 & 521 & 331 & 7.6 & 4.9 \\
\hline Case 5 & 41.91 & 45.55 & 413 & 468 & 6.1 & 4.4 \\
\hline Case 6 & 50.22 & 0 & 377 & 407 & 6.3 & 6 \\
\hline Case 7 & 120.33 & 26.85 & 622 & 369 & 17.5 & 7.6 \\
\hline
\end{tabular}

Abbreviations: CRT, central retinal thickness; IVR, intravitreal ranibizumab; pre, preintravitreal ranibizumab; post, postintravitreal ranibizumab; VEGF, vascular endothelial growth factor.

Table 4 Correlation between VEGF and CRT or flare at the time of additional IVR treatment

\begin{tabular}{lll}
\hline $\begin{array}{l}\text { Item of } \\
\text { correlation }\end{array}$ & $\begin{array}{l}\text { Coefficient of } \\
\text { correlation with VEGF }\end{array}$ & P-value \\
\hline Flare & 0.65 & 0.12 \\
CRT & 0.73 & 0.06 \\
\hline
\end{tabular}

Abbreviations: CRT, central retinal thickness; IVR, intravitreal ranibizumab; VEGF, vascular endothelial growth factor. 
study, this issue should be investigated further in future in studies with a large sample size.

Additionally, in this study, case 4 showed improved CRT, even though VEGF and flare values had worsened from baseline to the time of additional IVR. In case 2, CRT had worsened, even though VEGF and flare had decreased. Case 6 showed no improvement in CRT, even though VEGF had decreased to 0 and flare had improved. Therefore, additional exacerbating factors, other than VEGF, are probably involved in CME. In the report by Noma et al, ${ }^{7}$ factors involved in the exacerbation of CME included inflammatory molecules, such as IL-6, ICAM-1, MCP-1, and PlGF, in addition to VEGF. In future, whether inhibition of these molecules could be therapeutic should be investigated.

This study had some limitations. Flare in patients with intraocular lens implants and those with cataract was considered to be similar. These groups were not investigated separately in the present study, and no report on this matter was found in the literature. In future, the study should be repeated in such groups of patients, separately.

\section{Conclusion}

Our study suggests that in patients with untreated BRVO, flare may be effectively used for the estimation of VEGF in the aqueous humor, prior to IVR treatment. We show that IVR brought about a significant improvement in patients with BRVO, but CME recurred at a high frequency.
Inflammation-related molecules other than VEGF might be associated with this recurrence.

\section{Acknowledgment}

This study was presented at the 120th Annual Meeting of the Japanese Ophthalmological Society: Efficacy of intravitreal ranibizumab injection to branch retinal vein occlusion.

\section{Disclosure}

The authors report no conflicts of interest in this work.

\section{References}

1. Miyake K, Miyake T, Kayazawa F. Blood-aqueous barrier in eyes with retinal vein occlusion. Ophthalmology. 1992;99(6):906-910.

2. [No authors listed] Argon laser photocoagulation for macular edema in branch vein occlusion. The Branch Vein Occlusion Study Group. Am J Ophthalmol. 1984;98(3):271-282.

3. Noma H, Mimura T, Tatsugawa M, Shimada K. Aqueous flare and inflammatory factors in macular edema with central retinal vein occlusion. BMC Ophthalmol. 2013;13:78.

4. Noma H, Minamoto A, Funatsu H, et al. Intravitreal levels of vascular endothelial growth factor and interleukin- 6 are correlated with macular edema in branch retinal vein occlusion. Graefes Arch Clin Exp Ophthalmol. 2006;244(3):309-315.

5. Thach AB, Yau L, Hoang C, Tuomi L. Time to clinically significant visual acuity gains after ranibizumab treatment for retinal vein occlusion: BRAVO and CRUISE trials. Opthalmology. 2014;121(5):1059-1066.

6. Ito Y, Saishin Y, Sawada O, et al. Comparison of single injection and three monthly injections of intravitreal bevacizumab for macular edema associated with branch retinal vein occlusion. Clin Opthalmol. 2015;9:175-180.

7. Noma H, Mimura T, Eguchi S. Association of inflammatory factors with macular edema in branch retinal vein occlusion. JAMA Ophthalmol. 2013;131(2):160-165.
Clinical Ophthalmology

\section{Publish your work in this journal}

Clinical Ophthalmology is an international, peer-reviewed journal covering all subspecialties within ophthalmology. Key topics include: Optometry; Visual science; Pharmacology and drug therapy in eye diseases; Basic Sciences; Primary and Secondary eye care; Patient Safety and Quality of Care Improvements. This journal is indexed on

\section{Dovepress}

PubMed Central and CAS, and is the official journal of The Society of Clinical Ophthalmology (SCO). The manuscript management system is completely online and includes a very quick and fair peer-review system, which is all easy to use. Visit http://www.dovepress.com/ testimonials.php to read real quotes from published authors. 